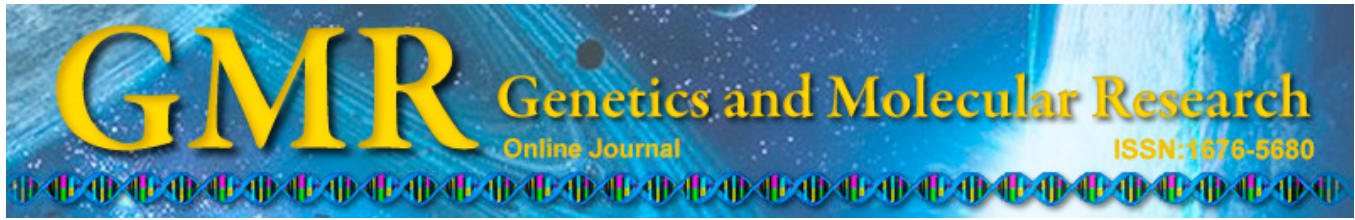

\title{
Evaluation of the genetic diversity of Bupleurum using amplified fragment length polymorphism analysis
}

\author{
S.Y. Ke ${ }^{1 *}$, L.L. Shi ${ }^{2 *}$, Y.Z. Ma ${ }^{1}$ and X.J. Zhou $^{3}$ \\ ${ }^{1}$ Faculty of Life Science, Tangshan Normal University, Tangshan, Hebei, \\ China \\ ${ }^{2}$ College of Biological Sciences and Biotechnology, Beijing Forestry University, \\ Beijing, China \\ ${ }^{3}$ Beijing Center for Physical and Chemical Analysis, Beijing, China \\ *These authors contributed equally to this study. \\ Corresponding author: L.L. Shi \\ E-mail: linglingshi2005@163.com
}

Genet. Mol. Res. 14 (1): 2590-2599 (2015)

Received March 13, 2014

Accepted June 3, 2014

Published March 30, 2015

DOI http://dx.doi.org/10.4238/2015.March.30.18

\begin{abstract}
Radix bupleuri (Chaihu), the dried root of the Bupleurum plant, is an important component of traditional Chinese medicine. In this study, we examined the genetic diversity of 11 Bupleurum strains, originating from 7 provinces in China, using amplified fragment length polymorphism analysis. A total of 274 polymorphic bands were obtained using 6 primer combinations, indicating a high level of polymorphism across all strains. An estimation of the relative relationships among strains revealed genetic distances ranging from 0.2183 to 0.7372 , with an average of 0.4161 . The 2 most closely related varieties were Bupleurum chinense DC. strains collected from Lushi, Henan, and Zhangjiakou, Hebei, with a genetic nearness of 0.2183 . Hierarchical clustering divided the strains into 3 main groups, with $B$. falcatum L. from Hebei and Liaoning Provinces forming a cluster that diverged from
\end{abstract}


that of B. smithii Wolff. and B. chinense DC. B. falcatum L. (Sandao chaihu), collected from Heze, Shandong, clustered independently of the other strains, suggesting that this strain may have been introduced from a different location or that it arose as a result of intraspecific variation. B. smithii Wolff. (Hei chaihu) was closely associated with B. scorzonerifolium Willd. (Nan chaihu) and B. chinense DC. (Bei chaihu), suggesting a common genetic origin.

Key words: Amplified fragment length polymorphism; Bupleurum; Chaihu; Radix bupleuri; Genetic diversity; Phylogenetic analysis

\section{INTRODUCTION}

Radix bupleuri (Chaihu) is the dried root of the Bupleurum plant and an important component of traditional Chinese medicine. It is listed in the official Chinese Pharmacopoeia for the treatment of influenza, fever, malaria, hepatitis, jaundice, nephritis, dizziness, bitter taste in the mouth, lung diseases, cancer, menstrual disorders, and infections (Yu, 1999; Zhao et al., 2009). Chemical analyses have revealed a wide array of important compounds present in $R$. bupleuri, including saponins, flavonoids, coumarins, fatty acids, steroids, polysaccharides, and polyacetylenes (Yu, 1999). Among these, the saponins have been identified as the major bioactive compounds and are used as a chemical standard for the evaluation of $R$. bupleuri potency (Liu et al., 2002; Tzeng et al., 2012).

As described in the Chinese Pharmacopoeia, $R$. bupleuri is derived from the dried roots of either Bupleurum chinense DC. (Bei chaihu) or B. scorzonerifolium Willd. (Nan chaihu). However, in addition to these authentic sources of $R$. bupleuri, there are more than 20 other Bupleurum species that are habitually used as a source of $R$. bupleuri, including $B$. smithii Wolff. (Hei Chaihu) and B. falcatum L. (Sandao chaihu). Due to the growing demand for $R$. bupleuri, the limited natural sources of Bupleurum plants have been depleted rapidly, leading to a sharp increase in commercial cultivation. Regional diversity, both within and between Bupleurum species, has led to uncertainty in the quality of $R$. bupleuri, with significant implications regarding the medicinal use of this plant. Furthermore, the genetic relationship within the germplasm remains poorly understood, limiting the ability of growers to improve commercial strains.

Molecular markers provide an efficient tool for evaluating genetic diversity and phylogeny at the DNA level without having to rely on complex pedigree records, environmental conditions, or epistatic and pleiotropic effects (Seefelder et al., 2000; Tao et al., 2009). Various types of molecular analyses, including restriction fragment length polymorphism, random amplified polymorphic DNA (RAPD), and amplified fragment length polymorphism (AFLP) have been successfully used to assess the genetic diversity of plant species (Morgante and Olivieri, 1993; Meudt and Clarke, 2007). AFLP emerged as one of the most popular marker systems because it covers a large percentage of the genome and produces more independent polymorphic loci in a single analysis than other marker systems (Papa et al., 2005; Sathyanarayana et al., 2011; Kim et al., 2013).

In this paper, we used AFLP to analyze 11 strains of Bupleurum, primarily from northern China. These analyses will provide insight into the genetic relationship of Bupleurum germplasm resources and may allow for better utilization of $R$. bupleuri as a medical product. 


\section{MATERIAL AND METHODS}

\section{Plant materials}

A total of 11 Bupleurum strains were collected from the major R. bupleuri-producing regions of China. Individual plants were selected as representatives of distinct geographical locations across 7 provinces, primarily from northern China (Table 1).

\begin{tabular}{|c|c|c|c|c|c|}
\hline ID & Local name & Species & Location of origin & Latitude (E) & Longitude $(\mathrm{N})$ \\
\hline 1 & Hei chaihu & Bupleurum smithii Wolff. & Weinan, Shaanxi & $34^{\circ} 13^{\prime} 48^{\prime \prime}$ & $108^{\circ} 50^{\prime} 12^{\prime \prime}$ \\
\hline 2 & Bei chaihu & B. chinense DC. & Zhangjiakou, Hebei & $40^{\circ} 30^{\prime} 39^{\prime \prime}$ & $114^{\circ} 50^{\prime} 24^{\prime \prime}$ \\
\hline 3 & No. 31 chaihu & B. falcatum $\mathrm{L}$. & Anguo, Hebei & $38^{\circ} 24^{\prime} 12^{\prime \prime}$ & $115^{\circ} 20^{\prime} 48^{\prime \prime}$ \\
\hline 4 & Hei chaihu & B. smithii Wolff. & Longxi, Gansu & $34^{\circ} 98^{\prime} 20^{\prime \prime}$ & $104^{\circ} 61^{\prime} 25^{\prime \prime}$ \\
\hline 5 & Bei chaihu & B. chinense DC. & Lushi, Henan & $34^{\circ} 06^{\prime} 12^{\prime \prime}$ & $111^{\circ} 03^{\prime} 40^{\prime \prime}$ \\
\hline 6 & No. 31 Huang chaihu & B. falcatum $\mathrm{L}$. & Shenyang, Liaoning & $41^{\circ} 12^{\prime} 30^{\prime \prime}$ & $123^{\circ} 25^{\prime} 11^{\prime \prime}$ \\
\hline 7 & No. 805 Bei chaihu & B. chinense DC. & Leling, Shandong & $37^{\circ} 44^{\prime} 00^{\prime \prime}$ & $117^{\circ} 12^{\prime} 00^{\prime \prime}$ \\
\hline 8 & Sandao chaihu & B. falcatum $\mathrm{L}$. & Heze, Shandong & $31^{\circ} 50^{\prime} 41^{\prime \prime}$ & $115^{\circ} 48^{\prime} 50^{\prime \prime}$ \\
\hline 9 & Japan No. 31 chaihu & B. falcatum $\mathrm{L}$. & Anguo, Hebei & $38^{\circ} 40^{\prime} 62^{\prime \prime}$ & $115^{\circ} 33^{\prime} 17^{\prime \prime}$ \\
\hline 10 & Hei chaihu & B. smithii Wolff. & Shaodong, Hunan & $27^{\circ} 25^{\prime} 12^{\prime \prime}$ & $111^{\circ} 73^{\prime} 26^{\prime \prime}$ \\
\hline 11 & Nan chaihu & B. scorzonerifolium Willd. & Lintao, Gansu & $35^{\circ} 22^{\prime} 32^{\prime \prime}$ & $103^{\circ} 51^{\prime} 22^{\prime \prime}$ \\
\hline
\end{tabular}

\section{DNA extraction}

The improved cetyltrimethylammonium bromide (CTAB) method was used to extract genomic DNA from Bupleurum sporophytes. Young healthy leaves ( $\sim 0.1 \mathrm{~g}$ fresh weight) were ground into powder in liquid nitrogen using a pre-cooled mortar, transferred to $2-\mathrm{mL}$ centrifuge tubes, resuspended in $800 \mu \mathrm{L}$ preheated CTAB extraction buffer [containing 100 $\mathrm{mM}$ Tris- $\mathrm{HCl}, \mathrm{pH} 8.0,2 \% \mathrm{CTAB}(\mathrm{w} / \mathrm{v}), 20 \mathrm{mM}$ ethylenediamine-tetraacetic acid (EDTA), $1.4 \mathrm{M} \mathrm{NaCl}$, and $0.2 \% \beta$-mercaptoethanol $(\mathrm{v} / \mathrm{v})]$, and incubated for $30 \mathrm{~min}$ at $65^{\circ} \mathrm{C}$. Leaf mixtures were gently mixed at regular intervals during the course of incubation. Homogenates were then centrifuged for $10 \mathrm{~min}$ at $12,000 \mathrm{rpm}$ at room temperature. The supernatant was transferred to a fresh 2-mL tube containing an equal volume of chloroform and centrifuged at $12,000 \mathrm{rpm}$ for $10 \mathrm{~min}$. The protein extraction step was repeated until no white impurities on the aqueous interface remained. DNA was precipitated by adding $100 \%$ ethanol to 2 volumes of supernatant, followed by centrifugation at $6,000 \mathrm{rpm}$ for $10 \mathrm{~min}$. Then, the DNA pellet was washed with $70 \%$ ethanol, dried at room temperature, and dissolved in $0.2 \mathrm{~mL}$ TE buffer $(10$ $\mathrm{mM}$ Tris, $\mathrm{pH}$ 8.0, $1 \mathrm{mM}$ EDTA). The solution was incubated overnight at $37^{\circ} \mathrm{C}$ with the addition of $5 \mu \mathrm{L}$ RNase $(10 \mathrm{mg} / \mathrm{mL}$; Tiangen, China) and then extracted with an equal volume of chloroform until the middle layer disappeared. DNA was precipitated by the addition of $50 \mu \mathrm{L}$ $\mathrm{NaAc}, \mathrm{pH} 5.2$, and $100 \%$ ethanol to 2 volumes of supernatant and centrifuged at $6,000 \mathrm{rpm}$ for $10 \mathrm{~min}$. The residue was washed with $70 \%$ ethanol twice, dried at room temperature, and dissolved in $200 \mu \mathrm{L}$ TE buffer.

\section{AFLP reactions}

The AFLP method was performed as described by Vos et al. (1995), with minor modifications. Genomic DNA (generally 1-2 $\mu \mathrm{g}$ ) was digested with $1 \mu \mathrm{L}$ of each EcoRI [20 U/ $\mu \mathrm{L}$; New England Biolabs (NEB), USA)] and $M s e \mathrm{I}(10 \mathrm{U} / \mu \mathrm{L}$; NEB) in a final volume of $50 \mu \mathrm{L}$ 
containing $5 \mu \mathrm{L}$ 10X NEB buffer 4 and $0.5 \mu \mathrm{L}$ bovine serum albumin $(10 \mathrm{mg} / \mathrm{mL})$. After mixing, DNA samples were incubated at $37^{\circ} \mathrm{C}$ overnight and then at $65^{\circ} \mathrm{C}$ for 20 min to denature the enzymes. Ligation was performed by mixing $10 \mu \mathrm{L}$ digested DNA, $1 \mu \mathrm{L}$ EcoRI-adapter ( 5 $\mu \mathrm{M}$; Invitrogen, USA), $1 \mu \mathrm{L}$ MseI-adapter (50 $\mu \mathrm{M}$; Invitrogen), $1 \mu \mathrm{L}$ T4 DNA ligase (400 U/ $\mu \mathrm{L}$; NEB), and $2 \mu \mathrm{L}$ T4 DNA ligase buffer (NEB) in a final volume of $20 \mu \mathrm{L}$. The mixture was then incubated at $16^{\circ} \mathrm{C}$ overnight, followed by $65^{\circ} \mathrm{C}$ incubation for $10 \mathrm{~min}$. A pre-selective polymerase chain reaction (PCR) mixture contained $8 \mu \mathrm{L}$ ligated DNA, 4.0 $\mu \mathrm{L}$ 10X Ex Taq buffer (TaKaRa, Japan), 3.2 $\mu \mathrm{L}$ deoxyribonucleoside triphosphates (dNTPs) $(2.5 \mathrm{mM}$; TaKa$\mathrm{Ra}), 1 \mu \mathrm{L}$ Ex Taq polymerase (5 U/ $\mu \mathrm{L}$; TaKaRa), $1 \mu \mathrm{L}$ EcoRI adapter-directed primer (E-00, 10 $\mu \mathrm{M}$; Invitrogen), and $1 \mu \mathrm{L} \mathrm{MseI} \mathrm{adapter-directed} \mathrm{primer} \mathrm{(M-00,} 10 \mu \mathrm{M}$; Invitrogen) to yield a total volume of $40 \mu \mathrm{L}$. Pre-amplification was performed under the following conditions: $94^{\circ} \mathrm{C}$ for $2 \mathrm{~min} ; 30$ cycles of $94^{\circ} \mathrm{C}$ for $20 \mathrm{~s}, 56^{\circ} \mathrm{C}$ for $30 \mathrm{~s}$, and $72^{\circ} \mathrm{C}$ for $2 \mathrm{~min} ; 72^{\circ} \mathrm{C}$ for $10 \mathrm{~min}$; and holding at $4^{\circ} \mathrm{C}$. After checking for the presence of fragments $100-1000 \mathrm{bp}$ in length by agarose electrophoresis, the PCR product mixture was diluted 10 -fold with distilled water and used as a template for selective PCR amplification. Six pairs of fluorescently labeled EcoRI-MseI primers were used: E-AAC/M-CAT, E-AAC/M-CAG, E-ACA/M-CTT, E-ACA/M-CTA, EAGG/M-CAA, and E-ATC/M-CTC. A selective amplification reaction mixture contained 3 $\mu \mathrm{L}$ pre-selective PCR product (diluted 10-fold with distilled water), $2.0 \mu \mathrm{L} 10 \mathrm{X}$ Ex Taq buffer (TaKaRa), $0.5 \mu \mathrm{L}$ dNTPs (10 mM; TaKaRa), $0.5 \mu \mathrm{L}$ Ex Taq polymerase (5 U/ $\mu \mathrm{L}$; TaKaRa), 1 $\mu \mathrm{L}$ EcoRI-primer (100 $\mu \mathrm{M}$; Invitrogen), and $1 \mu \mathrm{L}$ MseI-primer (100 $\mu \mathrm{M}$; Invitrogen) to yield a total volume of $20 \mu \mathrm{L}$. Selective amplification was performed with the following touchdown PCR profile: $94^{\circ} \mathrm{C}$ for $5 \mathrm{~min} ; 13$ cycles of $94^{\circ} \mathrm{C}$ for $30 \mathrm{~s}, 65^{\circ} \mathrm{C}$ for $30 \mathrm{~s}$, and $72^{\circ} \mathrm{C}$ for $1 \mathrm{~min}$, with the annealing temperature decreasing by $0.7^{\circ} \mathrm{C}$ per cycle; 30 cycles of $94^{\circ} \mathrm{C}$ for $30 \mathrm{~s}$, $56^{\circ} \mathrm{C}$ for $30 \mathrm{~s}$, and $72^{\circ} \mathrm{C}$ for $1 \mathrm{~min}$; an extension at $72^{\circ} \mathrm{C}$ for $10 \mathrm{~min}$; and holding at $4^{\circ} \mathrm{C}$. The sequences of AFLP adaptors and selected primer pairs are shown in Table 2. The resulting PCR products, which produced smeared bands primarily distributed in the $100-500$ bp range on agarose gel electrophoresis, were used for capillary electrophoresis in the next experiment.

Table 2. Sequences of pre-amplification and selective amplification primers for amplified fragment length polymorphism (AFLP) analysis.

\begin{tabular}{ll}
\hline Name & Sequence $\left(5^{\prime} \rightarrow 3^{\prime}\right)$ \\
\hline EcoRI-adapter & \\
E-F & CTCGTAGACTGCGTACC \\
E-R & AATTGGTACGCAGTCTAC \\
MseI-adapter & GACGATGAGTCCTGAG \\
M-F & TACTCAGGACTCAT \\
M-R & \\
Pre-amplification primers & GACTGCGTACCAATTC \\
E-00 & GATGAGTCCTGAGTAA \\
M-00 & \\
Selective amplification primers & GACTGCGTACCAATTCAAC \\
E-AAC & GACTGCGTACCAATCACA \\
E-ACA & GACTGCGTACCAATCAGG \\
E-AGG & GACTGCGTACCAATTCATC \\
E-ATC & GATGAGTCCTGAGTAACAT \\
M-CAT & GATGAGTCCTGAGTAACAG \\
M-CAG & GATGAGTCCTGAGTAACTT \\
M-CTT & GATGAGTCCTGAGTAACTA \\
M-CTA & GATGAGTCCTGAGTAACAA \\
M-CAA & GATGAGTCCTGAGTAACTC \\
M-CTC & \\
\hline
\end{tabular}




\section{Data analysis}

Data regarding selectively amplified DNA fragments were separated by capillary electrophoresis using an ABI3730xl DNA Analyzer (Applied Biosystems). The polymorphic AFLP loci were analyzed using GeneMapper, version 4.0, with the LIZ 600 size standard (Applied Biosystems). Polymorphic AFLP fragments were scored as present (1) or absent (0) based on the AFLP pattern amplified by each primer combination. The ability of the most informative primer pairs to differentiate among strains was assessed by calculating the percentage of polymorphic bands and the resolving power (Rp) (Prevost and Wilkinson, 1998). Rp has been described to correlate strongly with the ability to distinguish between species according to the following formula (Gilbert et al., 1999; Chennaoui-Kourda et al., 2012):

$$
\mathrm{R}_{\mathrm{p}}=\sum \mathrm{Ib}
$$

(Equation 1)

where $I b=1-(2 \times|0.5-\mathrm{p}|)$ and $p$ is the proportion of species containing the 1 band. POPGENE 1.3.1 was used to calculate the genetic diversity. Genetic distances were calculated using the formula of Nei and $\mathrm{Li}$ (1979). The obtained matrix was then used for cluster analysis assessed by unweighted pair group mean of arithmetic method analysis (UPGMA) implemented in the NTSYS-pc software (version 2.1) (Rohlf, 2000).

\section{RESULTS}

\section{Evaluation of primer combinations}

High-quality genomic DNA is the key to a successful AFLP reaction. Accordingly, we used the improved CTAB method described in the Material and Methods section for all genomic DNA extraction. The DNA quality was further supported by subsequent experiments, including restriction endonuclease digestion, ligation of adaptors, and PCR.

Prior to EcoRI/MseI digestion, we performed a pre-amplification step using primers E-00 and M-00, followed by a selective-amplification using primers $\mathrm{E}+3$ and $\mathrm{M}+3$. Using a variety of reaction conditions, we were able to identify 6 AFLP primer combinations with selective bases at the 3 '-end of each primer. These primer combinations were then tested to determine both the quality of AFLP fingerprinting and the levels of polymorphism for each pair (Figure 1). Together, these 6 AFLP primer combinations generated a total of 276 bands, the vast majority of which (274) were polymorphic (Table 3 ). The number of bands varied considerably between primer pairs, ranging from as low as 31 for primers E-ACA and M-CTT to as high as 65 for primers E-ATC and M-CTC. The average numbers of total and polymorphic bands per primer pair were 46 and 45.7, respectively. Rp values ranged from 4.51 to 6.57 with a collective value of 34.14 (Table 3). E-ATC and M-CTC showed the highest Rp and were the most powerful primer combination for demonstrating DNA genetic diversity among the analyzed species. Together, these results validate the use of AFLP as a tool for examining the genetic diversity of Bupleurum. 


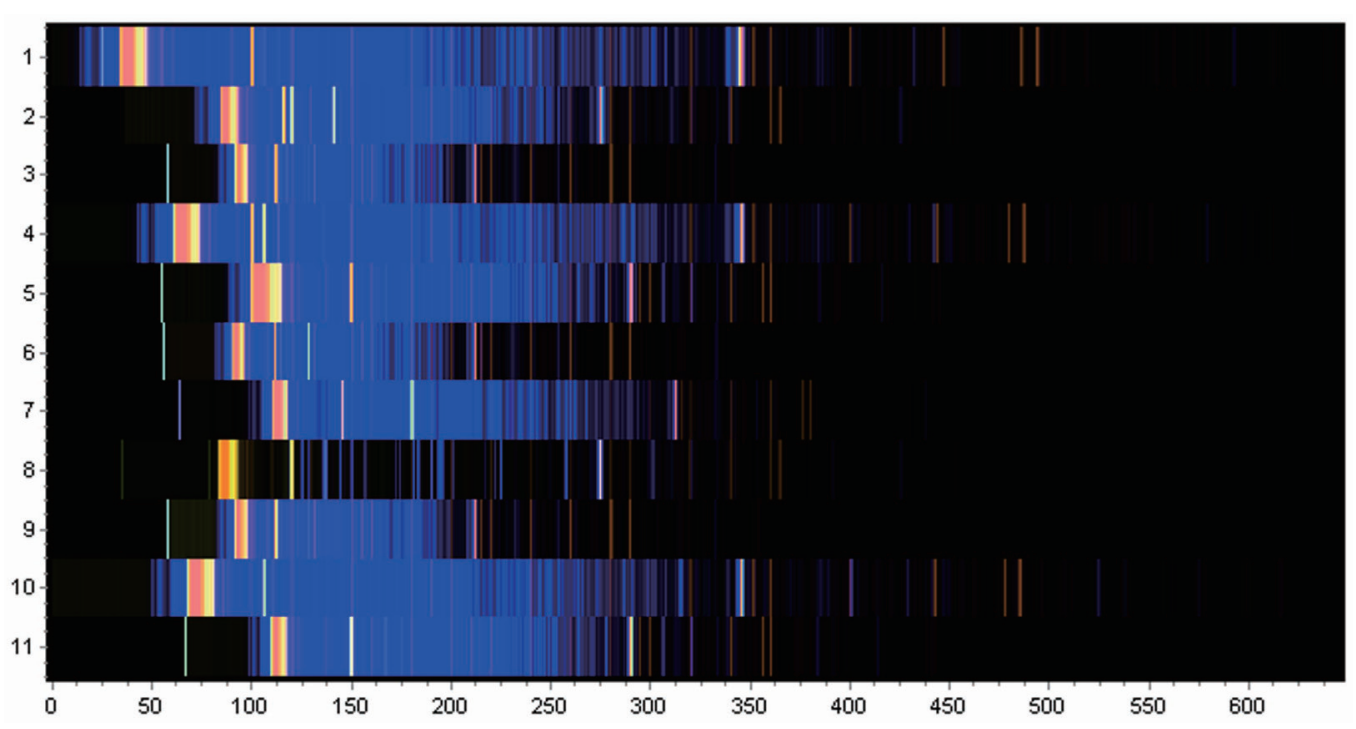

Figure 1. Amplification products of the primer pair E-AGG and M-CAA across 11 Bupleurum strains. Codes 1-11 represent all individuals sampled in Table 1 in order.

Table 3. Percentage of polymorphic bands and resolving power of 6 AFLP primer combinations among 11 Bupleurum strains.

\begin{tabular}{lcccc}
\hline Primer combination & \multicolumn{2}{c}{ No. of AFLP-PCR bands scored } & Resolving power (Rp) \\
\cline { 2 - 4 } & Total & Polymorphic & $\%$ & 4.0 \\
\hline E-AAC/M-CAT & 41 & 41 & 100.0 & 6.85 \\
E-AAC/M-CAG & 40 & 40 & 100.0 & 4.79 \\
E-ACA/M-CTT & 31 & 31 & 98.4 & 6.13 \\
E-ACA/M-CTA & 64 & 63 & 97.1 & 5.29 \\
E-AGG/M-CAA & 35 & 34 & 100.0 & 6.57 \\
E-ATC/M-CTC & 65 & 65 & & 34.14 \\
Total & 276 & 274 & \\
\hline
\end{tabular}

\section{AFLP DNA fingerprinting}

Next, the 274 polymorphic bands identified by AFLP analysis were used to construct DNA fingerprints. Banding patterns from each of the 6 primer pairs could be used to differentiate between strains. From these, the 10 most reproducible and reliable AFLP bands (with band sizes of 209, 204, 192,187, 163,161,149,120,107, and $101 \mathrm{bp)} \mathrm{generated} \mathrm{by} \mathrm{the} \mathrm{primer} \mathrm{pair}$ E-AAC and M-CAT were used for the construction of AFLP DNA fingerprints for each of the 11 Bupleurum strains (Figure 2). Figure 2 clearly shows that, using this method, each strain exhibited a unique fingerprint and was easily distinguished from the others. These results provide a useful method for the identification of Bupleurum genotypes at the DNA level and may also have practical implications on the breeding and clinical application of $R$. bupleuri. 


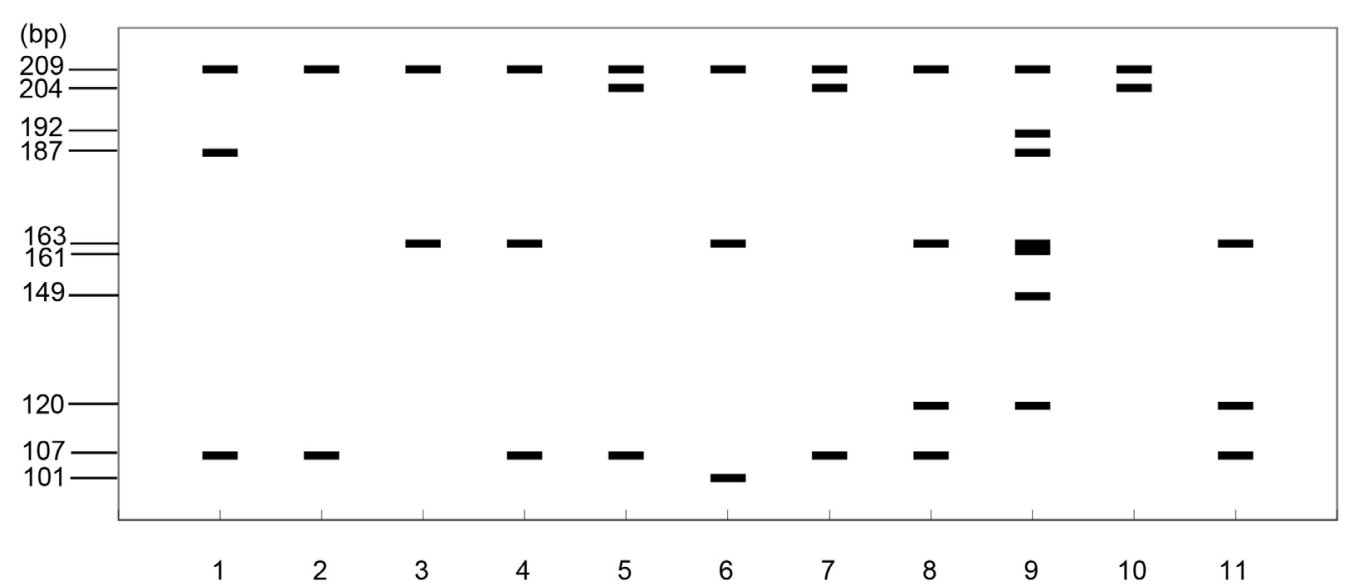

Figure 2. Amplified fragment length polymorphism DNA fingerprints of 11 Bupleurum strains. Codes 1-11 represent all individuals sampled in Table 1 in order.

\section{AFLP diversity and species relationships}

In order to estimate the phylogenetic relationship among Bupleurum strains, the polymorphic AFLP primer fragments were analyzed using the Gendist program, according to the formula of Nei and Li (1979). The resulting genetic distance matrix revealed values ranging from 0.2183 to 0.7372 , with an average distance of 0.4161 (Table 4), indicating a relatively high degree of genetic diversity at the target DNA sequences. The highest divergence was seen between strains B. falcatum L. and B. smithii Wolff., which were collected from Anguo, Hebei, and Longxi, Gansu, respectively. The smallest divergence was observed between the 2 B. chinense DC. strains collected from Lushi, Henan, and Zhangjiakou, Hebei, respectively.

\begin{tabular}{|c|c|c|c|c|c|c|c|c|c|c|c|}
\hline ID & 1 & 2 & 3 & 4 & 5 & 6 & 7 & 8 & 9 & 10 & 11 \\
\hline 1 & $* * * *$ & 0.7255 & 0.6078 & 0.7686 & 0.7255 & 0.5843 & 0.7569 & 0.6627 & 0.5216 & 0.7882 & 0.7333 \\
\hline 2 & 0.3209 & $* * * *$ & 0.5686 & 0.7765 & 0.8039 & 0.5529 & 0.7961 & 0.6471 & 0.5137 & 0.7804 & 0.7804 \\
\hline 3 & 0.4978 & 0.5645 & $* * * *$ & 0.5961 & 0.5529 & 0.7961 & 0.5922 & 0.6392 & 0.6784 & 0.6314 & 0.6000 \\
\hline 4 & 0.2631 & 0.2530 & 0.5174 & $* * * *$ & 0.7529 & 0.5490 & 0.7529 & 0.6902 & 0.4784 & 0.7608 & 0.7843 \\
\hline 5 & 0.3209 & 0.2183 & 0.5925 & 0.2838 & $* * * *$ & 0.5294 & 0.7882 & 0.6784 & 0.4824 & 0.8039 & 0.7569 \\
\hline 6 & 0.5373 & 0.5925 & 0.2281 & 0.5996 & 0.6360 & $* * * *$ & 0.5608 & 0.6235 & 0.7176 & 0.6000 & 0.5843 \\
\hline 7 & 0.2786 & 0.2281 & 0.5240 & 0.2838 & 0.2380 & 0.5784 & $* * * *$ & 0.6863 & 0.4902 & 0.8039 & 0.7647 \\
\hline 8 & 0.4114 & 0.4353 & 0.4475 & 0.3708 & 0.3880 & 0.4724 & 0.3765 & $* * * *$ & 0.5922 & 0.7255 & 0.7176 \\
\hline 9 & 0.6509 & 0.6661 & 0.3880 & 0.7372 & 0.7291 & 0.3318 & 0.7129 & 0.5240 & $* * * *$ & 0.5608 & 0.5137 \\
\hline 10 & 0.2380 & 0.2480 & 0.4599 & 0.2734 & 0.2183 & 0.5108 & 0.2183 & 0.3209 & 0.5784 & $* * * *$ & 0.7961 \\
\hline 11 & 0.3102 & 0.2480 & 0.5108 & 0.2429 & 0.2786 & 0.5373 & 0.2683 & 0.3318 & 0.6661 & 0.2281 & $* * * *$ \\
\hline
\end{tabular}

Bold values indicate the smallest and the largest genetic distances.

To further clarify the genetic relationship of the 11 Bupleurum species examined, the genetic distance values were subjected to hierarchical clustering using UPGMA. This type of clustering analysis is often used to identify species that are closely related to their geographical origins and cultivation history. UPGMA clustering arranged the 11 varieties into 3 groups (Figure 3). The first clade grouped 3 strains of B. falcatum L., 2 of which were collected from 
Anguo, Hebei, and 1 from Shenyang, Liaoning. The remaining 8 were divided into 2 major groups. The first group contained a single species, B. falcatum L., collected from Heze, Shandong, while the second group was further subdivided into 3 subsets. The first subset contained varieties B. scorzonerifolium Willd. collected from Lintao, Gansu, and B. smithii Wolff. collected from Longxi, Gansu. The second group consisted of another B. smithii Wolff. strain collected from Shaodong, Hunan, and 3 strains of $B$. chinense DC. collected from Leling, Shandong, Lushi, Henan, and Zhangjiakou, Hebei, respectively. The final subset consisted of a single B. smithii Wolff. strain collected from Weinan, Shaanxi.

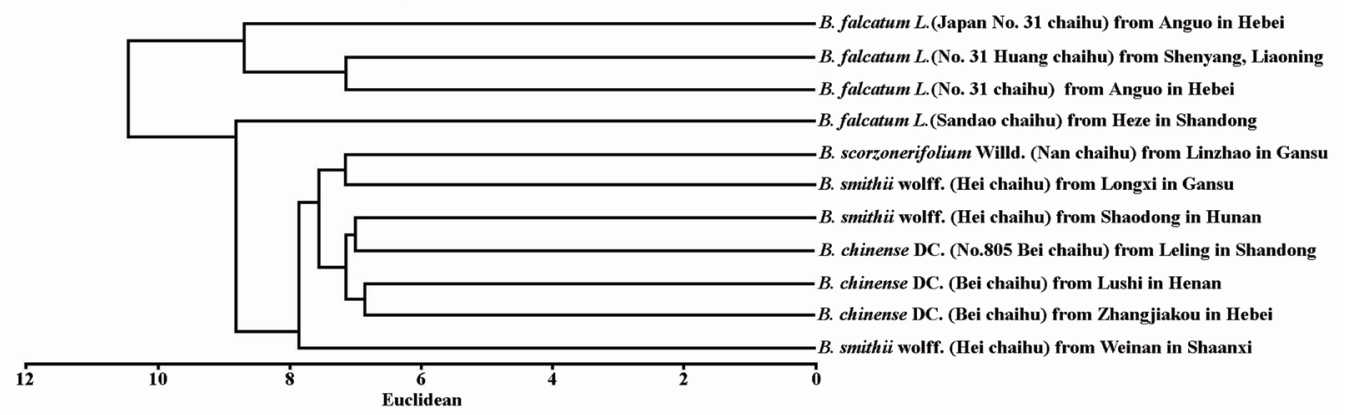

Figure 3. Interspecific analysis of Bupleurum species by unweighted pair group mean of arithmetic method.

It is noteworthy that the B. falcatum L. strains from Hebei and Liaoning Provinces readily clustered independently of the other Bupleurum species, B. smithii Wolff., B. scorzonerifolium Willd., and B. chinense DC. However, B. falcatum L. collected from Heze, Shandong, clustered independently of any other strains, suggesting that this strain may have been introduced from different sources in Japan or that a greater degree of intraspecific variation has occurred in this region. Another important relationship revealed by UPGMA clustering is the close association between $B$. smithii Wolff., B. scorzonerifolium Willd., and B. chinense DC., which is indicative of a relatively recent divergence. Finally, the close relationship observed between B. chinense DC. strains collected from Lushi, Henan, and Zhangjiakou, Hebei suggests a higher degree of similarity than would be expected from their relatively distant collection sites.

\section{DISCUSSION}

The primary goals of this study were to identify molecular markers suitable for the analysis of genetic diversity in Bupleurum and to improve our understanding of Bupleurum phylogeny. In this study, we identified a fingerprint of 10 genetic markers in Bupleurum that was capable of differentiating between both species and closely related strains.

The 10-marker fingerprint was pulled from a total of 276 AFLP generated using 6 distinct primer combinations. The high number of fragments generated in this analysis is greater than that of other molecular methods from similar species, including RAPD (Liang et al., 2002) and inter-simple sequence repeats (Zhan et al., 2010). Furthermore, the AFLP primer combinations that were used are characterized by a collective $\mathrm{Rp}$ rate of 34.14. Our data support the use of AFLP markers as a complementary tool for reliable Bupleurum phylogenetic 
construction and an alternative approach for estimating the molecular variability among these species, consistent with that seen in other species (Sciacca et al., 2010; Chennaoui-Kourda et al., 2012; Kim et al., 2013).

The E-ATC/M-CTC primer combination exhibited the highest Rp value of any primer combination examined in this study. These primers have previously been used to examine the genetic diversity of many of the same species described here (Chennaoui-Kourda et al., 2012). An average of 46 markers per primer combination was observed for each of the 6 primer combinations used, suggesting a relatively high degree of genetic diversity at the DNA level. This assumption is strongly supported by genetic distance analyses, with distances ranging from 0.2183 to 0.7372 , which indicates high genetic diversity within Bupleurum. These data will provide a useful tool for the cultivation and management of these medicinal strains.

Hierarchical clustering of Bupleurum strains was also performed using genetic distance values. Strains of B. falcatum L. from Hebei and Liaoning Provinces were readily distinguished from those of other species. This clear divergence was not surprising because $B$. falcatum L. originated in Japan and has only recently been introduced in China. However, $B$. falcatum L., which was collected from Heze, Shandong, clustered independent of any other B. falcatum L. strain, suggesting that this strain may have originated from a different part of Japan or that intraspecific variation has occurred in the region where this strain was collected; similar events have also been observed in other species (Sánchez-Gómez et al., 2010; Groot et al., 2012). This substantial divergence observed within B. falcatum L. highlights the difficulty of traditional variety identification using phenotypes alone. The addition of molecular markers could help determine not only the source of some plants but also provide information necessary for the identification and optimization of plant varieties (Urbanelli et al., 2007; Abdul Wahab et al., 2014).

The close relationship that was observed between $B$. smithii Wolff., B. scorzonerifolium Willd., and B. chinense DC. is indicative of a common genetic origin, possibly due to a lack of diversity within the gene pool. Furthermore, we can assume that the analyzed species have evolved from a common ancestor of B. smithii Wolff. in or around Weinan, Shaanxi. Finally, the close relationship among $B$. chinense DC. strains collected from Lushi, Henan, and Zhangjiakou, Hebei, is noteworthy given the large distance and diverse climates separating these 2 locations.

The data derived from this study will be useful for the establishment of uniform quality standards for $R$. bupleuri and for the development of rational management strategies for clinical use. In the meantime, it will be necessary to combine phenotypic and molecular approaches to identify significant correlations between molecular markers and agronomic parameters for identifying and characterizing Bupleurum species. These measures are necessary to ensure the quality of medicinal compounds that serve as the core of traditional Chinese medicinal practices.

\section{ACKNOWLEDGMENTS}

Research financially supported by the subitem of the Science and Technology Ministry of China (\#2011BAI07B05-4), the Major Science and Technology Project of Tangshan (\#12130203A), and the Ph.D. Program Foundation of Tangshan Normal University (\#11A01). We also wish to thank Deqiang Zhang and Qingzhang Du for their help with image analysis, and Qiangshi Wang and Yun Chu for their technical support. 


\section{REFERENCES}

Abdul Wahab AA, Taj-Aldeen SJ, Hagen F, Diophode S, et al. (2014). Genotypic diversity of Pseudomonas aeruginosa in cystic fibrosis siblings in Qatar using AFLP fingerprinting. Eur. J. Clin. Microbiol. Infect. Dis. 33: 265-271.

Chennaoui-Kourda H, Marghali S, Zitouna N and Trifi-Farah N (2012). Phylogenetic relationships of Mediterranean Hedysarea species assessed by AFLP markers. Plant Syst. Evol. 298: 51-58.

Gilbert JE, Lewis RV, Wilkinson MJ and Caligari PDS (1999). Developing an appropriate strategy to assess genetic variability in plant germplasm collections. Theor. Appl. Genet. 98: 1125-1131.

Groot GA, Verduyn B, Wubs ERJ, Erkens RHJ, et al. (2012). Inter- and intraspecific variation in fern mating systems after long-distance colonization: the importance of selfing. BMC Plant Biol. 12: 3.

Kim SH, Lee JS, Lee GJ, Kim JS, et al. (2013). Analyses of genetic diversity and relationships in four Calanthe taxa native to Korea using AFLP markers. Hort. Environ. Biotechnol. 54: 148-155.

Liang ZT, Qin MJ, Wang ZT, Huang Y, et al. (2002). Identification of Bupleurum L. plants by RAPD technology. Zhongcaoyao 33: 1117-1119.

Liu QX, Tan L, Bai YJ, Liang H, et al. (2002). A survey of the studies on saponins from Bupleurum in past 10 years. Zhongguo Zhong Yao Za Zhi 27: 7-11.

Meudt M and Clarke AC (2007). Almost forgotten or latest practice? AFLP applications, analyses and advances. Trends Plant Sci. 12: 106-117.

Morgante M and Olivieri AM (1993). PCR-amplified microsatellites as markers in plant genetics. Plant J. 3: 175-182.

Nei M and Li WH (1979). Mathematical model for studying genetic variation in terms of restriction endonucleases. Proc. Natl. Acad. Sci. U. S. A. 76: 5269-5273.

Papa R, Troggio M, Ajmone-Marsan P and Nonnis Marzano F (2005). An improved protocol for the production of AFLP markers in complex genomes by means of capillary electrophoresis. J. Anim. Breed. Genet. 122: 62-68.

Prevost A and Wilkinson MJ (1998). A new system of comparing PCR primers applied to ISSR fingerprinting of potato cultivars. Theor. Appl. Genet. 98: 107-112.

Rohlf FJ (2000). NTSYSpc. Numerical Taxonomy and Multivariate Analysis System - Version 2.10b. Applied Biostatistics Inc., New York.

Sánchez-Gómez D, Majada J, Alía R, Feito I, et al. (2010). Intraspecific variation in growth and allocation patterns in seedlings of Pinus pinaster Ait. submitted to contrasting watering regimes: can water availability explain regional variation? Ann. For. Sci. 67: 504-505.

Sathyanarayana N, Leelambika M, Mahesh S and Jaheer M (2011). AFLP assessment of genetic diversity among Indian Mucuna accessions. Physiol. Mol. Biol. Plants 17: 171-180.

Sciacca F, Fichera C, Di Silvestro S, Conte E, et al. (2010). Genetic diversity of durum wheat as determined by AFLP in fluorescence. Biol. Plant. 54: 198-200.

Seefelder S, Ehrmaier H, Schweizer G and Seigner E (2000). Male and female genetic linkage map of hops, Humulus lupulus L. Plant Breed. 119: 249-255.

Tao J, Ai YW, Luo YQ, Yang L, et al. (2009). AFLP analysis of genetic variation of Hyphantria cunea (Drury) populations in Beijing and a nearby site. For. Stud. China 11: 14-19.

Tzeng TF, Lu HJ, Liou SS, Chang CJ, et al. (2012). Vinegar-baked Radix bupleuri regulates lipid disorders via a pathway dependent on peroxisome-proliferator-activated receptor- $\alpha$ in high-fat-diet-induced obese rats. Evid. Based Complement. Alternat. Med. 2012: 827278.

Urbanelli S, Della Rosa V, Punelli F, Porretta D, et al. (2007). DNA-fingerprinting (AFLP and RFLP) for genotypic identification in species of the Pleurotus eryngii complex. Appl. Microbiol. Biotechnol. 74: 592-600.

Vos P, Hogers R, Bleeker M, Reijans M, et al. (1995). AFLP: a new technique for DNA fingerprinting. Nucleic Acids Res. 23: 4407-4414.

Yu J (1999). Advances in the study on medicament portions of Chaihu. J. Chin. Med. Mater. 22: 315-317.

Zhan QQ, Sui C, Wei JH, Fan SC, et al. (2010). Construction of genetic linkage map of Bupleurum chinense DC. using ISSR and SSR markers. Yao Xue Xue Bao 45: 517-523.

Zhao RZ, Liu SJ, Mao SR and Wang YJ (2009). Study on liver targeting effect of vinegar-baked Radix bupleuri on resveratrol in mice. J. Ethnopharmacol. 126: 415-420. 